\title{
Statistical approach to identify Naples city's real driving cycle referring to the Worldwide harmonized Light duty Test Cycle (WLTC) framework
}

\author{
L. Della Ragione \& G. Meccariello \\ Istituto Motori Consiglio Nazionale delle Ricerche, Italy
}

\begin{abstract}
In the context of sustainability, which requires an interdisciplinary approach, evaluation of car emissions and strategies for their reductions plays a fundamental role. Any sustainable private transport solutions could be proposed solely from the integration of many disciplines, architects, environmentalists, policy makers, and so it may be addressed with different approaches. These solutions would be applied at different geographical levels, i.e. national, regional or urban scale. In addition, it is fundamental to keep in mind that nowadays, the new Worldwide harmonized Light vehicles Test Procedures (WLTP) and Worldwide harmonized Light duty Test Cycle (WLTC) cycle will reproduce a situation closer to the reality with respect to the Extra Urban Driving Cycle (EUDC)/New European Driving Cycle (NEDC) driving cycle. During the research activities of the DRIVE IN ${ }^{2}$ project (DRIVEr monitoring: technologies, methodologies, and IN-vehicle INnovative systems for safe and eco-compatible driving), that involved universities, public research institutions and automobile companies, an experimental campaign was realized in the roads of Naples city. So, instrumented vehicles for the simultaneous acquisition of kinematic variables, localization and emissions data have been used. Until now, data are analyzed for each specific vehicle with the aim of investigating a correlation between vehicle kinematics and relative emissions of regulated pollutants. Here the attention will be focused on the global results strictly from a kinematic point of view. Therefore, we will present driving cycles representative of the real behaviour of cars on two typical city paths, one very flat and the other run over hill. Real driving emissions were acquired during the experimental campaign with Portable Emissions Measurement
\end{abstract}


System (PEMS) and precise geolocalization data were registered by Global Positioning System (GPS). The collected kinematic database was analyzed by multivariate statistical analysis to obtain a cluster of speed parts, each of them representative of a driving mode, start\&stop, cruise, acceleration, deceleration and the initial phase of a trip. Finally, applying some statistical criteria, and going in the same direction of a WLTC approach, a driving cycle composed of a succession of speed parts was built.

Keywords: statistical analysis, driving cycle, WLTC.

\section{Introduction}

The control of pollutant emissions and fuel consumption produced by a vehicle in an urban area are the more frequent requirements posed from the legislator for the protection of community health. To fulfil such requests, several studies needed to take into account critical operating conditions affecting the real pollutant emissions and fuel consumption of in-use vehicles. The problem is thus, in principle, defined when the following information is available:

- Road characteristics (number of lanes, type of pavements, crossing...)

- Traffic management rules (traffic lights, parking, speed limits...)

- Vehicle composition (fleet composition, vehicle age distribution...) and activity.

- Vehicle flow and density, congestion level of road.

During the development of the ARTEMIS project, new driving cycles, the Common Artemis Driving Cycle (CADC), utilized for emission testing on a chassis dynamometer, have been defined. They are capable of considering more attributes than the simple speed to characterize driving behaviour, not only in the determination of driving cycles but also in the emission modelling [1]. More recently, in-use emissions testing with PEMS became one of the solutions proposed to ensure that emissions are well controlled in the real use of passenger cars. Moreover, a new type approval procedure is going to be introduced by the European Commission, to take into account critical operating conditions affecting the real pollutant emissions and fuel consumption of in-use vehicles and not considered during the laboratory tests. The new procedure involves the monitoring of real driving emissions (RDE) on the road by a PEMS. In addition, it is fundamental to keep in mind that nowadays, the new WLTP and WLTC cycle reproduce a situation closer to the reality respect to the EUDC/NEDC driving cycle. In $[2,3]$ some results are reported of AECC coming from tests over the NEDC, the CADC, the new Worldwide Light-duty Test Cycle (WLTC - the test cycle for WLTP) and a set of cycles produced by a Random Cycle Generator. Emission measurements are performed on test bench and with (PEMS). During real driving over pre-selected routes, results show that there can be substantial differences for some pollutants measured as 'real driving emissions' (RDE) using PEMS equipment, compared to the test bench cycles in laboratory. Other results show the variations between technologies and the potential effectiveness of emissions control technologies evaluated on different test cycles. Also, the evaluation of nitrogen oxides (Nox) emissions is carried out in relation to the diesel 
car and to the current type-approval procedure tests [4]. This finding suggests that the current type-approval procedure does not adequately capture the on-road NOx emissions of diesel cars. Therefore, the added value to introduce complementary emissions test procedure that covers a wide range of normal operating conditions is underlined. With regard to the fuel consumption and carbon dioxide $\left(\mathrm{CO}_{2}\right)$ emissions, in [5] Makarchuk et al. stress out the test procedure, during which the vehicle is being driven along a defined speed profile, but mainly they highlight that the speed profile itself has a major impact on the resulting fuel consumption. They compare four important driving cycles, NEDC, WLTC, FTP75 and HWY, and assess the effect of engine operation in the cycle. The analysis shows that WLTC [7] offers the most homogeneous distribution of all in the analyzed cycles. The Joint Research Centre (JRC) [6], during the development of WLTP, has tested many gasoline and diesel vehicles, on both the current European type approval test procedure (NEDC) and the progressive versions of the WLTP. Their findings, to be considered as an initial and qualitative indication of the trends, demonstrated some differences between $\mathrm{CO}_{2}$ emissions over the NEDC and WLTP. Results from this study can be considered indicative of emission patterns of modern technology vehicles. During the development of ARTEMIS project, a new statistical approach has been proposed in Istituto Motori [8, 9], capable of considering more attributes than the simple speed to characterize driving behaviour, not only in the determination of driving cycles but also in the emission modelling. In this new framework many research programs have been carried out on this subject, whose aim was to determine driving behaviour and emission trends. Results show that if we consider a specific road, driving behaviour changes and so driving cycles of different characteristics always occur. In this paper, some findings regarding the real in use driving cycle determination are reported. During the research activities of the DRIVE IN ${ }^{2}$ project, that involved universities, public research institutions and automobiles company, an experimental campaign was realized in the roads of Naples city. So, instrumented vehicles for the simultaneous acquisition of kinematic variables, localization and emissions data have been used.

\section{Experimental activity}

Experimental activity was carried out with several vehicles with different engine displacement and type approvals. The vehicle list and global characteristics are shown in Table 1.

They are driven along two tracks of Naples characterized by a different road gradient. So the experimental campaign and data acquisition during the execution of the on road tests are carried out in two areas different for the topography of the streets, but that present very similar vehicular.

The first pattern is predominantly flat and a length of about $22 \mathrm{~km}$ (as shown in Fig. 1(a)), the second on the hilly area of Naples with varied terrain and sudden changes of slope of about $6 \mathrm{~km}$, includes positive $(+2.9 \%)$ and negative $(-3.6 \%)$ road gradient (as shown in Fig. 1(b)). Exhaust emissions of carbon monoxide 
Table 1: Vehicle characteristics.

\begin{tabular}{|l|c|c|c|}
\hline \multicolumn{1}{|c|}{ Vehicles } & Euro & Fuel type & $\begin{array}{c}\text { Engine displacement } \\
\left(\mathrm{cm}^{3}\right)\end{array}$ \\
\hline Fiat 500 & 5 & Gasoline & 900 \\
\hline Fiat Panda Bifuel & 4 & Gasoline/Metano & 1200 \\
\hline Fiat Panda Twin Air & 4 & Gasoline & 900 \\
\hline Jeep Renegade & 6 & Diesel & 1956 \\
\hline Fiat Lancia & $5 \mathrm{~A}$ & Gasoline & 1598 \\
\hline Fiat 500L & 6 & Gasoline/GPL & 1400 \\
\hline Citroen Jumper & 5 & Diesel & 2198 \\
\hline
\end{tabular}

(CO), total hydrocarbons (THC), (NOx), $\mathrm{CO}_{2}$ were acquired on road by using a PEMS connected also to the Engine Control Unit for saving the main engine parameters and to the GPS for the geographical coordinates and altitude.

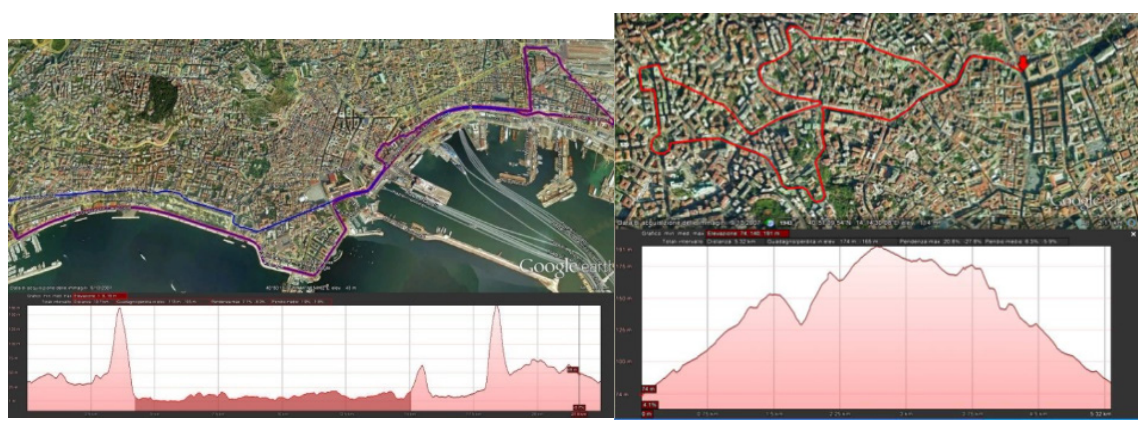

(a)

(b)

Figure 1: Experimental route: (a) Outward (Istituto Motori-P.za Garibaldi) and return (P.za Garibaldi-Istituto Motori); (b) the hilly area of Naples (Google Earth).

\subsection{Experimental setup}

Vehicles have been instrumented for on-road tests (as shown in Figure 2).

The main components of the monitoring system used in this work are:

- a Semtech gas analyzer produced by Sensor to measure at $1 \mathrm{~Hz} \mathrm{CO}, \mathrm{NOx}$ and $\mathrm{CO}_{2}$ emissions. This analyser uses NDIR cell (Non-Dispersive Infrared) for $\mathrm{CO}$ and $\mathrm{CO}_{2}$ measurements, NDUV cell for nitric oxide (NO)/nitrogen dioxide $\left(\mathrm{NO}_{2}\right)$ and separate electrochemical sensor for oxygen. The analyser is calibrated on a regular basis and zeroes itself on start-up using outside air.

- an EFM (Exhaust Flow Meter) by Sensor. 
- $\quad$ an On Board Diagnostics (OBD) interface and logging computer running proprietary software Engine Data Scan (EDS) to acquire engine operating parameters (speed, rpm, engine air flow...).

- a GPS receiver by Racelogic Ltd to acquire the spatial position.

- a video camera to record traffic situations.

The signals from all devices have been synchronized by using the same information obtained from different sources (i.e. speed from GPS and OBD). The emission measurements acquired by PEMS and the kinematics and GPS data, are filtered, synchronized and analyzed using statistical methods.

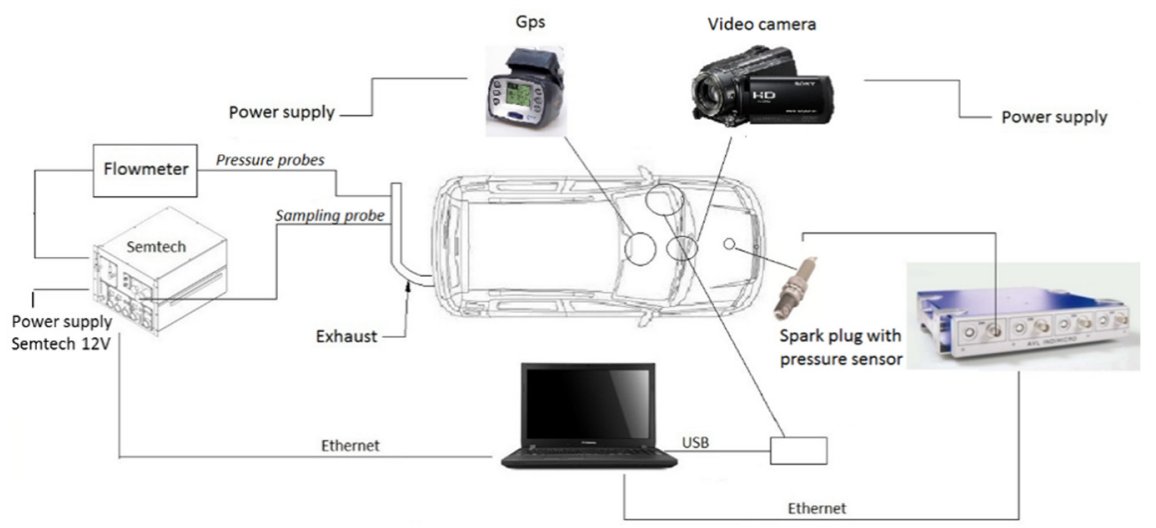

Figure 2: On-board installation.

The whole experimental campaign realized by the seven cars is made up of fifth-one trips carried out during two times of the day, morning and afternoon rush hours, and with two different drivers. Finally, a huge database of in use kinematic, GPS and emission data is built and analyzed.

\section{Kinematic driving cycle analysis}

The approach proposed in this paper for the determination of driving cycles is based on the analysis of road data by statistical methods to determine typical and statistically representative groups of driving cycles, engine operating conditions and emission correlated profiles. Driving behaviours are relative to different road networks, traffic conditions and specific features of each geographical area, with vehicles of different segments and technology. They constitute global sets from which representative pieces are chosen to build a driving cycle going in the same direction of the WLTC framework.

Following the general approach, the velocity profile is segmented in a succession of sequences, so that a sequence is the part of motion of a vehicle between two successive stops. Driving cycles have been determined without any conditioning of data with respect to road network, but keeping the information detected on road, in terms of GPS coordinates latitude, longitude and altitude. The 
method utilized to determine the driving cycles is based on sequence characterization. Variables describing each sequence are relative to speed and acceleration, sequence time and length, and relative frequency distribution.

Table 2: $\quad$ Variables characterizing kinematic DC.

\begin{tabular}{|c|c|}
\hline Variable & Description \\
\hline $\mathrm{mv}(\mathrm{km} / \mathrm{h})$ & Mean of running speed $(\mathrm{v}>0)]$ \\
\hline $\mathrm{mv} 2\left(\mathrm{~km}^{2} / \mathrm{h}^{2}\right)$ & Mean of square speed $(v>0)$ \\
\hline $\operatorname{mv} 3\left(\mathrm{~km}^{3} / \mathrm{h}^{3}\right)$ & Mean of cube speed $(\mathrm{v}>0)$ \\
\hline Tral (s) & idling time $\mathrm{v}=0$ in second \\
\hline Trunning (s) & total running time $(\mathrm{v}>0)$ in second \\
\hline Dist (m) & distance covered \\
\hline Time(s) & Total duration of the sequence $(\mathrm{s})$ \\
\hline m_vapos $\left(\mathrm{m}^{2} / \mathrm{s}^{3}\right)$ & $\begin{array}{l}\text { Mean of instantaneous values of product }(a(t) \bullet v(t)) \text { when } \\
v(t)>0 \text { and } a(t)>0\end{array}$ \\
\hline V20 (\%) & $\%$ time speed $<20$ \\
\hline V30 (\%) & $\%$ time $20<$ speed $<30$ \\
\hline V40 (\%) & $\%$ time $30<$ speed $<40$ \\
\hline V60 (\%) & $\%$ time $40<$ speed $<60$ \\
\hline $\mathrm{V} 100(\%$ & $\%$ time speed $>60$ \\
\hline Paccel1 (\%) & $\%$ time with acceleration in range $[-\infty ;-1.4] \mathrm{m} / \mathrm{s}^{2}$ \\
\hline Paccel2 (\%) & $\%$ time with acceleration in range $[-1.4 ;-0.6] \mathrm{m} / \mathrm{s}^{2}$ \\
\hline Paccel3 (\%) & $\%$ time with acceleration in range $[-0.6 ;-0.2] \mathrm{m} / \mathrm{s}^{2}$ \\
\hline Paccel4 (\%) & $\%$ time with acceleration in range $[-0.2 ;+0.2] \mathrm{m} / \mathrm{s}^{2}$ \\
\hline Paccel5 (\%) & $\%$ time with acceleration in range $[+0.2 ;+0.6] \mathrm{m} / \mathrm{s}^{2}$ \\
\hline Paccel6 (\%) & $\%$ time with acceleration in range $[+0.6 ;+1.4] \mathrm{m} / \mathrm{s}^{2}$ \\
\hline Paccel7 (\%) & $\%$ time with acceleration in range $[+1.4 ;+\infty] \mathrm{m} / \mathrm{s}^{2}$ \\
\hline
\end{tabular}

Clustering of sequences by multivariate statistical analysis give the basic information to automatically separate driving cycles from the real velocity profile detected on the road. A new cycle starts when a sequence belonging to a different cluster is encountered in the car speed time series.

In the above Table 2, variables characterizing driving behaviour are reported. In terms of kinematic, they refer to speed and acceleration attributes, time duration and length of driving cycle, idling and driving time.

In the following, the whole experimental dataset is divided into two subsets, relating to the two paths. Statistical analysis results presented are relative to the path A. Anyway, in the results and discussion section, a driving cycle representative of the path B is shown. The road tests on Path A are subdivided in 1126 sequences on which Principal Component Analysis (PCA) and Cluster Analysis (CA) are performed, to better characterize sequences and classify them into homogeneous groups. Principal component analysis of a data matrix extracts the dominant patterns in the dataset making use of a complementary set of score and loading. The first results are shown in Figure 3, where comparing the loadings plot to the scores plot enables us to understand how the variables relate to the 
observations. In fact, in the plot they are simultaneously presented scores showing correlations between observations, and loadings showing correlations between variables. The scaling of the axis depends upon the pre-treatment of the data. The loadings plot has a scale of +1 to -1 .

The plot evidence that the different vehicle types and fuel does not affect the performance of the sequences that are strongly influenced by the traffic condition encountered during the trip. In fact, the kinematic variables used show a differentiated distribution of the sequences with respect to their kinematic structure. Some variables have a high value $(\max =1)$ that means that the component is aligned with the original variable, a few are close to zero and they have no influence on the general pattern of observation.

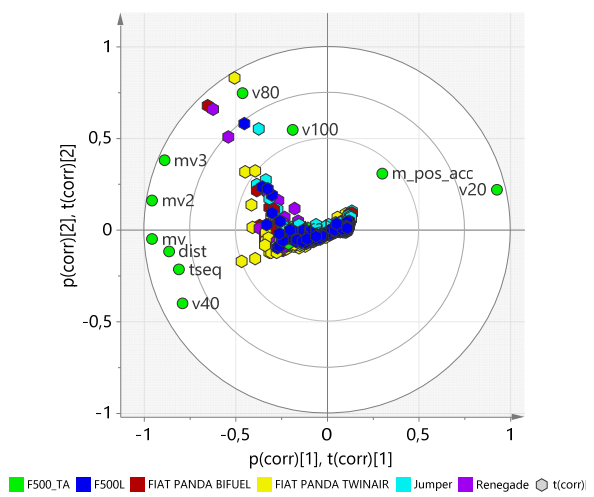

(a)

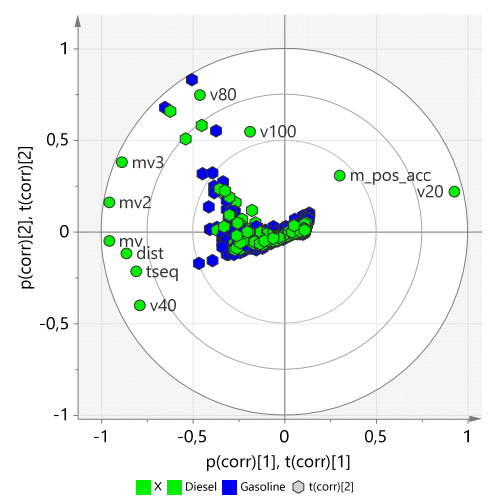

(b)

Figure 3: (PCA-X) - (a) Coloured according to model terms and vehicle. (b) Coloured according to model terms and to fuel type.

A low value (min -1) indicates an opposite influence. There is not a specific trend in the sequences (observations) influenced by the vehicles type or different fuel. In fact, the sequences cloud, coloured by fuel types, does not present specific clusters.

The entire set of sequences, that constitute the "speed parts", is grouped in five clusters applying cluster analysis on principal component variables. In Figure 4, an overlay of the representative speed part profiles of each cluster is shown.

Clustering of sequences by multivariate statistical analysis gives the basic information to cut automatically driving cycles from the real car velocity profile detected on the road. So driving cycles are defined starting from the sequence clusters. The rule used to define a cycle, defined as the succession of homogeneous sequences, is the following:

1. A cycle begins with the first sequence of a trip, or when there is a transition from one group to another, i.e. 1-2, 1-3, 2-3, and vice versa.

2. A cycle ends with the last sequence of a journey, or a transition with the previous sequence 
Following this rule, a sequence set forms 241 driving cycles. They are subsequently grouped into clusters, in a similar way as explained for sequences. Moreover, in this phase, new variables characterizing the driving cycles pattern are built and after the same multivariate statistical methodologies are carried out to investigate and summarize their specific characteristics.

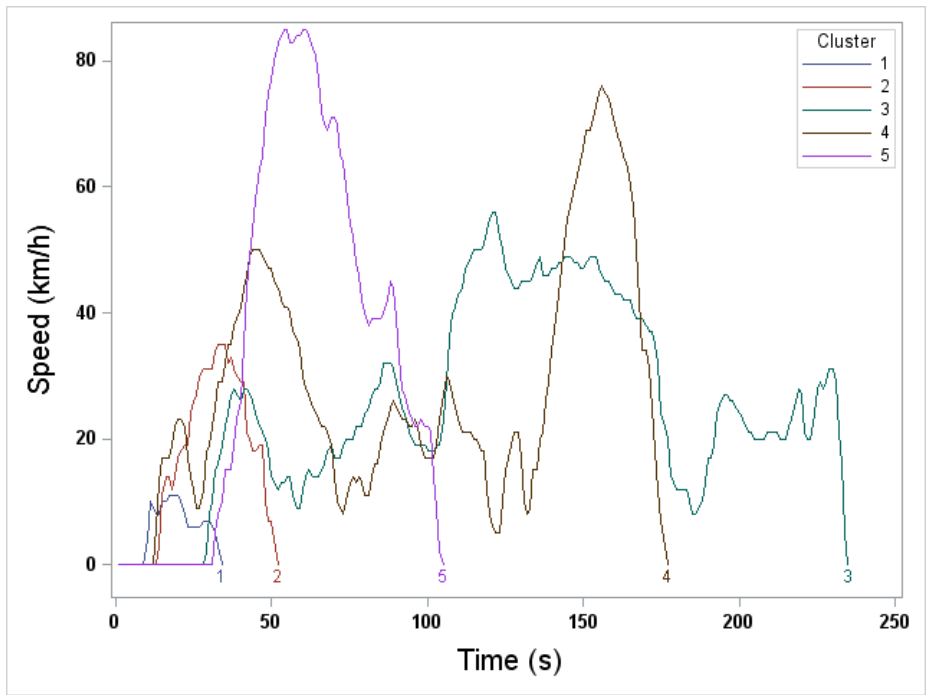

Figure 4: Speed profile overlay of representative sequences.

\section{Results and discussion}

Driving cycles are clustered into groups to determine typical and statistical car performance in different roads with different experienced traffic conditions. Cluster analysis performed on cycle variables, some of them reported in table 3, groups them into three well defined clusters. In such a way the different behaviour and traffic situations that have occurred are synthesized, and then the different driving styles are evidenced. For this reason, on each driving cycle (statistical unit) the average value of kinematic variables are calculated. Significant variables are: cycle mean velocity $(m v)$, cycle total idling time (tral), total distance covered (dist) and cycle total time $(t c y c)$.

Results are illustrated by cluster representation in the Can 1, Can 2 scatter plot (Figure 5), where each point stands for a driving cycle.

In Figure 5, Can 1 values are correlated with variables that differentiate the cluster's cycles in terms of total distance covered. Cluster 1-3 cycle distances are about the same (dist $\cong 1278-1290 \mathrm{~m}$ ) while cluster 2 value is about dist $\cong 1433 \mathrm{~m}$. Can 2 values differentiate them from slow $(\mathrm{mv} \cong 16-19 \mathrm{~km} / \mathrm{h})$ to fast $(\mathrm{mv} \cong 38 \mathrm{~km} / \mathrm{h})$ in terms of mean velocity. Moreover, they are sorted in decreasing mode for time duration and idling. 


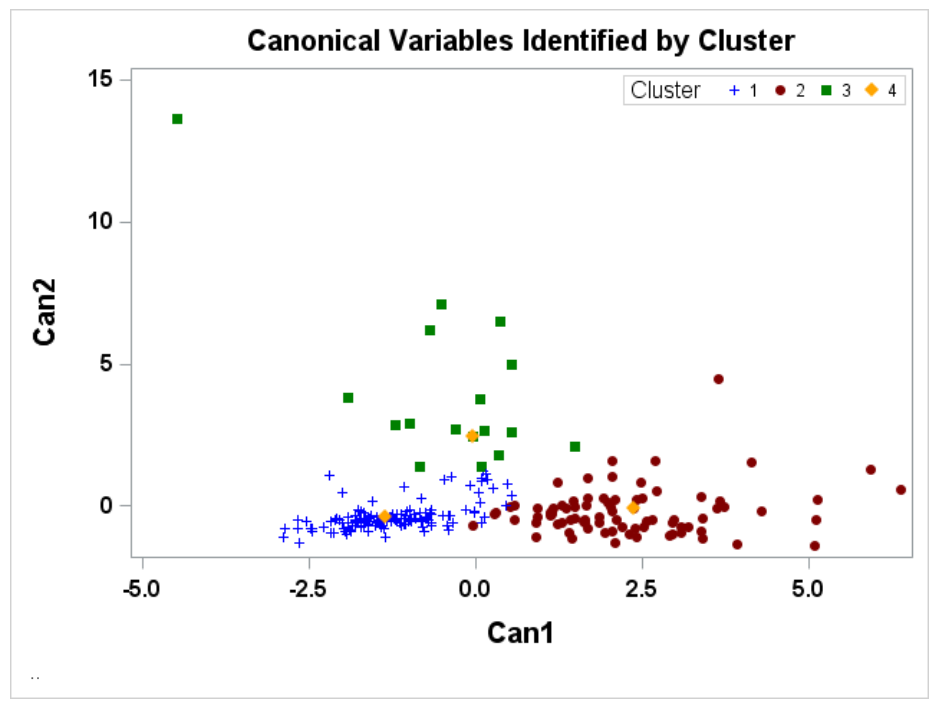

Figure 5: Cluster representation of cycle.

From each group the most representative cycle is determined by discriminant analysis. Therefore, in Figure 5, cluster 4 points stand for the representative elements of each cluster.

Table 3 explains that, for each cluster, mean values of variables most representative, so it is possible to point out fundamental differences in the kinematic features. In this way, results coming from Can1-Can2 plot are confirmed and more simple understood in terms of physical phenomena. Finally, the global cycle could be built by placing successively the most representative from each cluster, ordering according to the average speed, or by starting from cluster 1 to follow. This way could be considered as statistical criteria, but obviously, it has to be posed in a specific context, in the sense that it is necessary to visualize where it is realized during the path. However, it is specific for different kinematic conditions and summarizes information related to road/driver/traffic

Table 3: Cluster cycle mean characteristics.

\begin{tabular}{|l|l|c|c|c|}
\hline & CLUSTER & 1 & 2 & 3 \\
\hline N cycles & & 142 & 82 & 17 \\
\hline $\mathrm{mv}(\mathrm{km} / \mathrm{h})$ & $\begin{array}{l}\text { mean } \\
\text { speed }\end{array}$ & 16.79 & 19.60 & 37.79 \\
\hline tral $(\mathrm{sec})$ & idling time & 80.22 & 41.83 & 12.70 \\
\hline dist $(\mathrm{m})$ & $\begin{array}{l}\text { distance } \\
\text { covered }\end{array}$ & 1278.58 & 1433.81 & 1290.74 \\
\hline tcyc $(\mathrm{sec})$ & $\begin{array}{l}\text { cycle } \\
\text { duration }\end{array}$ & 317.28 & 236.47 & 141.88 \\
\hline
\end{tabular}


conditions more frequently experienced in real use. Therefore, the global cycles could have different average speeds, length and distance traveled, idling time, running time, distributions of gear use and acceleration/deceleration and so on. Figures 6 and 7 present the velocity profiles versus time of the global cycles built for Path A and Path B. Particularly in Figure 6, global driving cycle for Path A shown a mean velocity of about $22 \mathrm{~km} / \mathrm{h}$ and a distance covered of about 5460 meters. Instead, Path B shown a mean velocity of about $18 \mathrm{~km} / \mathrm{h}$ and a distance covered of about 2900 meters.

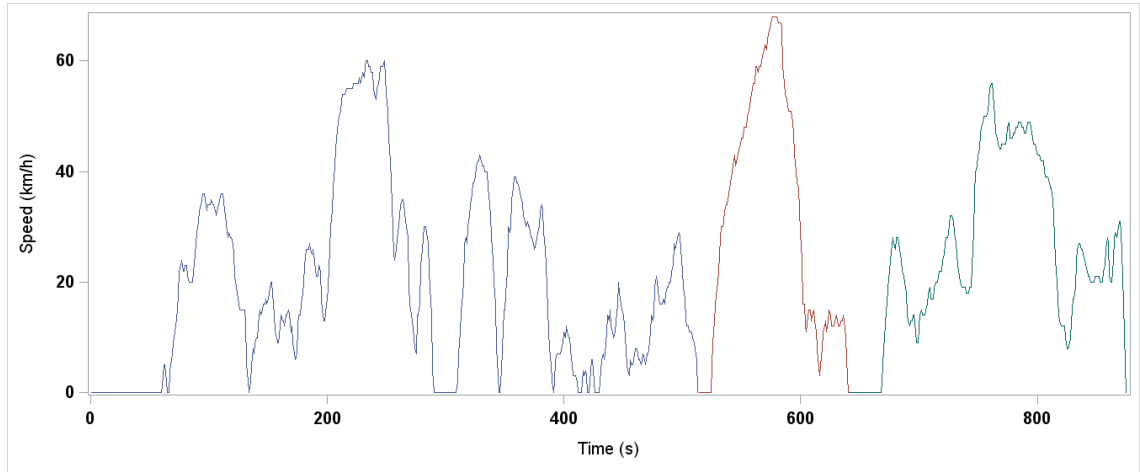

Figure 6: Typical cycle Path A.

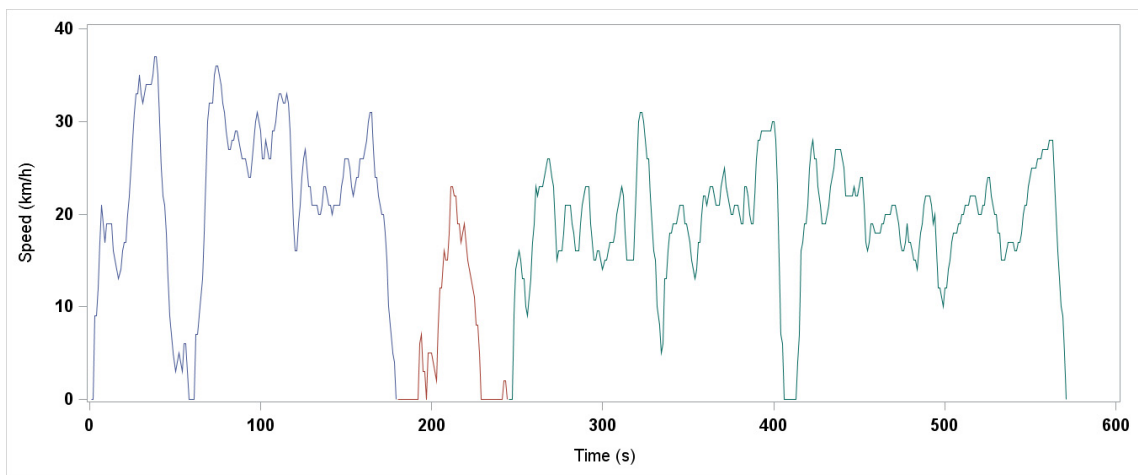

Figure 7: Typical cycle Path B.

\section{Conclusions}

In this paper some results regarding the real in use driving cycle determination are reported. During the research activities of the DRIVE IN 2 project, that involved universities, public research institutions and automobiles company, an experimental campaign was realized in the roads of Naples city, by different cars equipped with kinematic data acquisition, GPS recording and PEMS (Portable Emission Measurement Systems) equipment. So, instrumented vehicles for the 
simultaneous acquisition of kinematic variables, localization and emissions data have been used. Some results relative to kinematic data analysis are presented. An aspect under investigation is the fact that nowadays cars have a strong individual behaviour in the emission production, thus driving cycles must be as more representative of real use as possible. Resulting global driving cycles built for Path $\mathrm{A}$ and for Path B, however, are specific of different kinematic conditions and summarizes information related to road/driver/traffic conditions more frequently experienced in real use. Following this approach, the identified global cycles could have different kinematic characteristics in terms of average speeds, length and distance traveled, idling time, running time, distributions of gear use and acceleration/deceleration. Results regarding this activity obviously are a preliminary indication but also a strong starting point for reflection on the real representation of the cycles currently used for type approval, fuel consumption and emissions evaluation. Moreover, they could be useful to both policy makers and vehicle manufacturers in developing future emission policy/technology strategies.

\section{Acknowledgements}

This research has been realized within the project PON01_00744 DRIVE IN2, (DRIVEr Monitoring: Technologies, Methodologies, and IN-vehicle INnovative systems for a safe and eco-compatible driving), funded by (MIUR) PON "Ricerca e Competitività" 2007-2013 program.

\section{References}

[1] André M., Joumard R., Vidon R., Tassel P., Perret P., "Real-world European driving cycles, for measuring pollutant emissions from high- and lowpowered cars", Atmospheric Environment 40(31): 5944-5953, 2006.

[2] May J., Bosteels D., Favre C., "An Assessment of Emissions from Light-Duty Vehicles using PEMS and Chassis Dynamometer Testing," SAE Int. J. Engines 7(3):2014, doi: 10.4271/2014-01-1581.

[3] May J., Favre C., Bosteels D., "Emissions from Euro 3 to Euro 6 light-duty vehicles equipped with a range of emissions control technologies", Internal Combustion Engines: Performance, Fuel Economy and Emissions: IMechE, London, 2013.

[4] Weiss M., Bonnel P., Kühlwein J., Provenza A., Lambrecht U., Alessandrini S., Carriero M., Colombo R., Forni F., Lanappe G., Le Lijour, P., Manfredi U., Montigny F., Sculati M., "Will Euro 6 reduce the NOx emissions of new diesel cars? - Insights from on-road tests with Portable Emissions Measurement Systems (PEMS)", Atmospheric Environment 62:657-665, 2012.

[5] Makarchuk D., Kreicbergs J, Grislis A., Gailis M., “Analysis of energies and speed profiles of driving cycles for fuel consumption measurements", 14th International Scientific Conference Engineering for Rural Development, Latvia, 2015, ISSN 1691-5976. 
[6] Marotta A., Pavlovic J., Ciuffo B., Serra S., Fontaras G., “Gaseous Emissions from Light-Duty Vehicles: Moving from NEDC to the New WLTP Test Procedure", Environ. Sci. Technol. 2015, 49, 8315-8322, doi: 10.1021/acs.est.5b01364.

[7] Tutuianu M., Marotta A., Steven H., Ericsson E., Haniu T., Ichikawa N., Ishii H., Development of a World-wide Worldwide harmonized Light duty driving Test Cycle (WLTC) (Technical Report). UN/ECE/GRPE/WLTP-IG DHC subgroup, December 2013.

[8] Della Ragione L., Meccariello G., Prati M.V., "Statistical Investigation of In Use Emissions and Fuel Consumption Measured by PEM on Different Gasoline Cars", SAE Technical Paper 2013-01-1511, 2013, doi: 10.4271/2013-01-1511.

[9] Meccariello G., Della Ragione L., Prati M., Costagliola M. et al., "Real Time Emissive Behaviour of a Bi-Fuel Euro 4 SI Car in Naples Urban Area," SAE Int. J. Fuels Lubr. 6(3):959-967, 2013, doi: 10.4271/2013-24-0173. 